\title{
FRACTURA DE CADERA EN ADULTOS MAYORES: PREVALENCIA Y COSTOS EN DOS HOSPITALES. TABASCO, MÉXICO, 2009
}

\author{
Elsy del Carmen Quevedo-Tejero',a, Marco Antonio Zavala-González"1,b, \\ Arianna del Carmen Hernández-Gamas ${ }^{1, c}$, Hilda María Hernández-Ortega ${ }^{1, c}$
}

\begin{abstract}
RESUMEN
Objetivos. Determinar la prevalencia y costos directos de atención de fractura de cadera en adultos mayores derechohabientes usuarios de los hospitales de referencia del Instituto Mexicano del Seguro Social (IMSS) y de Petróleos Mexicanos (PEMEX) en Villahermosa, Tabasco, México, en el año 2009. Materiales y métodos. Estudio transversal. La información se basó en los registros de intervenciones quirúrgicas e informes institucionales de adultos mayores hospitalizados con registro de su atención.Se realizó análisis estadístico descriptivo considerando las variables: edad, sexo,tipo de fractura de cadera, mes de ocurrencia y costo directo de atención. Resultados.De 10765 registros de adultos mayores hospitalizados se encontró 57 casos de fractura de cadera (33 en el IMSS y 24 en PEMEX). La prevalencia de fractura de cadera fue 0,5\% (IMSS 1,1\% y PEMEX 0,3\%), siendo más frecuente en mujeres y en mayores de 69 años de edad. El tipo de fractura más frecuente fue en el cuello del fémur ( $78,9 \%$ del total). El costo estimado de atención médica hospitalaria por paciente fue de USD 5803 en el IMSS y USD 11800 en PEMEX. Conclusiones. La prevalencia de fractura de cadera fue mayor en los usuarios del IMSS. Los costos estimados de atención por paciente fueron mayores a los reportados en otras instituciones del sistema nacional de salud mexicano.
\end{abstract}

Palabras clave: Adulto mayor; Fracturas de cadera; Epidemiología descriptiva; Estudios retrospectivos; Costos y análisis de costo (fuente: DeCS BIREME).

\section{HIP FRACTURE IN OLDER ADULTS: PREVALENCE AND COSTS IN TWO HOSPITALS. TABASCO, MEXICO, 2009}

\begin{abstract}
Objectives. To determine hip fracture prevalence and direct healthcare costs in elderly users of the reference hospitals of the Mexican Institute of Social Insurance (IMSS by spanish initials) and Mexican Oils (PEMEX by spanish initials), from Villahermosa, Tabasco, Mexico, during 2009. Materials and methods. This is a cross-sectional study. The information was based on the registers of surgical interventions and institutional reports of the elderly inpatients who had a registered attention in their institution. Descriptive statistical analysis was performed considering the following variables: age, gender, hip fracture type, occurrence month, direct healthcare cost. Results. Out of 10,765 records of hospitalized elderly, 57 hip fracture cases were found (33 in the IMSS and 24 in PEMEX). Hip fracture prevalence was 0.5\%, (IMSS 1.1\% and PEMEX $0.3 \%$ ), being more frequent in women and older than 69 . The most frequent fracture type was the femur neck one (78.9\%). The estimated cost of healthcare in the hospital per patient was USD 5,803 in the IMSS and USD 11,800 in PEMEX. Conclusions. The hip fracture prevalence was higher in the IMSS users. Estimated healthcare costs per patient were higher than the reported in other institutions of the of the mexican health national system.
\end{abstract}

Key words: Elderly; Hip fractures; Epidemiology, descriptive; Retrospective studies; Costs and cost analysis (source: MeSH NLM).

\section{INTRODUCCIÓN}

El envejecimiento poblacional trae consigo repercusiones económicas, sanitarias y sociales que lo llevan a ser considerado un problema de salud pública ${ }^{(1)}$. En la década 1950-60 se integraron al grupo de personas adultas mayores cuatro millones de personas anualmente, mientras que en la primera década de este siglo esta cifra ascendió a más de quince millones (2). Esto es causado por la caída de la fecundidad, descenso de la mortalidad y movimiento migratorio ${ }^{(3)}$.

En México, se define como "adulto mayor" a toda persona de edad igual o mayor a 60 años ${ }^{(1-3)}$. Según el

\footnotetext{
División Académica de Ciencias de la Salud de la Universidad Juárez Autónoma de Tabasco. México.

a Médico, Maestra en Geriatría; ${ }^{b}$ Médico, Maestro en Educación; ' ${ }^{c}$ Estudiante de Medicina Humana.

* Resumen publicado en Libro de resúmenes del 14 Congreso de Investigación en Salud Pública. Cuernavaca, México: INSP; 2011.
}

Recibido: 01-04-11 Aprobado: 14-09-11 
Consejo Nacional de Población (CONAPO), en 2006 los adultos mayores representaban al $5,3 \%$ de la población, mientras que en el año 2009 esta proporción ascendió a $5,7 \%{ }^{(4,5)}$. Los estados mexicanos con mayor número de adultos mayores son Estado de México, Distrito Federal, Veracruz y Jalisco, mientras que los que tuvieron menor número en ese período fueron Baja California Sur, Quintana Roo y Colima ${ }^{(6)}$. En el Estado de Tabasco, en 2009 los adultos mayores representaban 4,7\% de la población. Al interior de este Estado, en el municipio de Centro (donde se ubica la ciudad capital), la proporción de adultos mayores pasó de 4,1\% en 2006 a $4,5 \%$ en 2009 , esperándose que llegue a $11,3 \%$ para el 2030 (4).

Considerando este panorama, resultan epidemiológicamente importantes los síndromes geriátricos, de entre los que destacan las caídas ${ }^{(7)}$, las cuales son un importante problema, dado que entre otras cosas, implica altos gastos en salud ${ }^{(8)}$. Se estima una prevalencia anual de $30 \%$, donde $10-15 \%$ resultan en una fractura ${ }^{(9-11)}$. Se calcula que mundialmente el número de fracturas de cadera aumentará a 2,6 millones para el 2025 y a 4,5 millones para el $2050^{(12)}$.

La incidencia de fracturas de cadera en mujeres crece exponencialmente con la edad ${ }^{(13)}$. Los tipos de fracturas más comunes son las del cuello del fémur, las pertrocantéreas y las trocantéreas ${ }^{(14)}$. Para que una caída resulte en una fractura de cadera, se requiere que se conjuguen factores como sexo femenino, debilidad muscular y baja densidad ósea ${ }^{(15-18)}$. La reparación quirúrgica es clave en la fractura de cadera, realizarla tempranamente parece no influir sobre la mortalidad, pero aporta grandes beneficios en términos de reducción del dolor, estancia intrahospitalaria y complicaciones ${ }^{(19)}$.

En México, se calcula que en el 2006 los costos del tratamiento de factura de cadera fueron de más de USD 97000 000,00 (20). El costo promedio de tratamiento quirúrgico de fractura de cadera en el Instituto $\mathrm{Na}$ cional de Rehabilitación, se estima de USD 1 729,85, incluyendo costos quirúrgicos, prótesis, nueve días de hospitalización y pruebas de laboratorio y rayos $\mathrm{X}$, antes y después de la cirugía ${ }^{(20)}$. Mientras que en el Seguro Popular, el costo aproximado es de USD 3 185,99, incluyendo consulta preoperatoria, intervención quirúrgica, material fungible, prótesis, dispositivos de fijación, medicamentos, exámenes de laboratorio y rayos $X{ }^{(20)}$.

En Tabasco, la mayoría de las unidades médicas de referencia se encuentran en Villahermosa, capital del Estado, encontrándose unidades de segundo y tercer nivel del Instituto Mexicano del Seguro Social (IMSS), el Instituto de Seguridad y Servicios Sociales de los Trabajadores del Estado (ISSSTE), la Secretaría de Salud (SS),
Petróleos Mexicanos (PEMEX) y la Secretaría de la Defensa Nacional (SEDENA) (21). Dadas las características socioeconómicas de Tabasco, la mayor proporción de su población es derechohabiente del IMSS y de los Servicios Médicos de PEMEX, ubicándose en Villahermosa, los hospitales de referencia de ambas instituciones: el Hospital General de Zona (HGZ) N. ${ }^{\circ} 46$ del IMSS y el Hospital Regional (HR) de los Servicios Médicos de PEMEX (21).

En el Estado de Tabasco, México, se desconocen múltiples datos sobre la fractura de cadera en adultos mayores: prevalencia, incidencia, causas, complicaciones, costos, entre otros aspectos. Considerando el contexto descrito, se decidió realizar una investigación con el objetivo de determinar la prevalencia y costos directos de atención de fractura de cadera en adultos mayores derechohabientes usuarios de los hospitales de referencia del IMSS y de PEMEX en Villahermosa, Tabasco, México, en el año 2009.

\section{MATERIALES Y MÉTODOS}

Se realizó una investigación transversal, en la que se estudiaron registros médicos de adultos mayores hospitalizados en el HGZ N ${ }^{\circ} 46$ del IMSS y el HR de PEMEX, en Villahermosa, Tabasco, durante el año 2009. Estudiándose las variables: edad, sexo, tipo de fractura de cadera, mes de ocurrencia y costos de atención.

Al ser agentes externos a las instituciones objeto de estudio y, por consiguiente, no estar inmersos en los procesos de atención médica en dichas instituciones, los autores realizaron las estimaciones basándose en fuentes secundarias, que fueron cuatro: 1) Informes institucionales de los departamentos de estadística del IMSS y de PEMEX, de donde se obtuvo el número de adultos mayores hospitalizados en las unidades médicas en cuestión durante el año referido; 2) Registros institucionales de intervenciones quirúrgicas, de donde se obtuvieron los datos referentes a las intervenciones quirúrgicas; 3) Acuerdo ACDO.SA3.HCT.240310/57.P.DF del $\mathrm{H}$. Consejo Técnico del IMSS ${ }^{(22)}$, documento normativo donde se indican los costos unitarios de atención por nivel en esta institución para los años lectivos 2009 y 2010; 4) Acuerdo PA-801-78300-4 de la Gerencia de Administración y Finanzas de PEMEX ${ }^{(23)}$, documento normativo donde se indican los costos unitarios de atención para el año lectivo 2009.

Previa autorización, se solicitó la información sobre el número de adultos mayores atendidos. Posteriormente, se consultó el número de intervenciones por fractura de cadera y los datos generales de los pacientes tratados 
Tabla 1. Casos de fractura de cadera: institución, edad, sexo y tipo de fractura.

\begin{tabular}{|c|c|c|c|c|c|c|c|c|c|c|c|c|c|c|c|c|c|c|c|c|c|c|}
\hline \multirow{4}{*}{$\begin{array}{l}\text { Fractura de } \\
\text { cadera }\end{array}$} & \multicolumn{10}{|c|}{$\mathrm{HGZ} \mathrm{N} .^{\circ} 46-$ IMSS } & \multicolumn{10}{|c|}{ HR - PEMEX } & \multirow{3}{*}{\multicolumn{2}{|c|}{$\begin{array}{l}\text { TOTAL } \\
(n=57)\end{array}$}} \\
\hline & \multicolumn{4}{|c|}{ 60-69 años } & \multicolumn{4}{|c|}{70 años y más } & \multirow{2}{*}{\multicolumn{2}{|c|}{ Total }} & \multicolumn{4}{|c|}{ 60-69 años } & \multicolumn{4}{|c|}{70 años y más } & \multirow{2}{*}{\multicolumn{2}{|c|}{ Total }} & & \\
\hline & $\mathrm{F}$ & $\mathrm{M}$ & Tc & tal & $\mathrm{F}$ & $M$ & & tal & & & $\mathrm{F}$ & $M$ & & tal & $\mathrm{F}$ & $\mathrm{M}$ & & tal & & & & \\
\hline & & & $\mathrm{n}$ & $\%$ & & & $\mathrm{n}$ & $\%$ & $\mathrm{n}$ & $\%$ & & & $\mathrm{n}$ & $\%$ & & & $\mathrm{n}$ & $\%$ & $\mathrm{n}$ & $\%$ & $n$ & $\%$ \\
\hline $\begin{array}{l}\text { Del cuello } \\
\text { del fémur }\end{array}$ & 4 & - & 4 & $(80)$ & 18 & 4 & 22 & (79) & 26 & (79) & 2 & 2 & 4 & (100) & 12 & 3 & 15 & (75) & 19 & $(79)$ & 45 & (79) \\
\hline Pertrocanteriana & - & 1 & 1 & (20) & 4 & 2 & 6 & (21) & 7 & (21) & - & - & - & - & - & 2 & 2 & (10) & 2 & (8) & 9 & (16) \\
\hline Subtrocanteriana & - & - & - & - & - & - & - & - & - & - & - & - & - & - & 2 & 1 & 3 & (15) & 3 & (13) & 3 & (5) \\
\hline Total & 4 & 1 & 5 & (100) & 22 & 6 & 28 & (100) & 33 & (100) & 2 & 2 & 4 & (100) & 14 & 6 & 20 & (100) & 24 & (100) & 57 & (100) \\
\hline
\end{tabular}

F: femenino; M: masculino; HGZ N. 46 - IMSS: Hospital General de Zona N. ${ }^{\circ} 46$ del Instituto Mexicano del Seguro Social; HR - PEMEX: Hospital Regional de los Servicios Médicos de Petróleos Mexicanos

por esta causa. Luego, se consultaron bases de datos hemerográficas para obtener un promedio de los días de estancia hospitalaria posterior a la intervención quirúrgica por fractura de cadera. Finalmente, se estimaron los costos directos de atención considerando como conceptos los costos unitarios de: estudios de radiodiagnósticos (uno pre- y otro transoperatorio), pruebas de laboratorio (biometría hemática y química sanguínea pre- y postoperatorias), intervención quirúrgica (reducción y osteosíntesis), días de hospitalización (nueve), honorarios médicos por día (nueve) y traslado en ambulancia al domicilio; de acuerdo con la Guía Clínica vigente ${ }^{(24)}$. Los datos fueron sistematizados y analizados usando Microsoft@ Excel@ versión 2003 para entorno Windows@)

La información fue analizada en tres etapas: 1) Estimación de la prevalencia en el período; 2) Obtención de distribución de frecuencias de casos según edad, sexo y mes de ocurrencia; 3) Estimación estandarizada de costos directos de atención desde la perspectiva del financiador, considerando los conceptos enunciados en el párrafo anterior. Los costos fueron obtenidos en pesos mexicanos (MXN) según la documentación consultada ${ }^{(22,23)}$, los que con fines de comparación con otros reportes fueron expresados en dólares americanos (USD) aplicando una tasa de cambio de MXN 1,00 = USD 0,0853.

\section{RESULTADOS}

\section{PREVALENCIA}

Se encontró que 2983 adultos mayores estuvieron hospitalizados en el HGZ No. 46 del IMSS y 7392 lo estuvieron en el HR de PEMEX, en Villahermosa, Tabasco, sumando 10765 adultos mayores. En estos, se encontró que 33 tuvieron diagnóstico de fractura de cadera en el IMSS y 24 en PEMEX, sumando 57 adultos mayores hospitalizados con dicho diagnóstico.
Al calcular estas cifras, se obtuvo una prevalencia de fractura de cadera de $11,1 \times 1000$ adultos mayores en el IMSS (1,1\%), y de 3,1 X 1000 en PEMEX (0,3\%), para sumar una prevalencia 5,3 X 1000 adultos mayores hospitalizados $(0,5 \%)$.

\section{DISTRIBUCIÓN DE FRECUENCIAS}

Se observó que la mayor proporción de casos de fractura de cadera ocurrieron en los pacientes de 70 años y más, siendo el sexo femenino el más afectado y las fracturas del cuello del fémur las más frecuentes. Tales resultados se observaron en ambas unidades médicas (Tabla 1). Al obtener la distribución de frecuencias de los casos de acuerdo al mes de ocurrencia, se observó que la mayoría de los casos ocurrieron en el mes de Diciembre, al igual que en el $\mathrm{HGZ} \mathrm{N}{ }^{\circ} 46$ del IMSS, mientras que en el HR de PEMEX ocurrieron en el mes de febrero (Figura 1).

\section{COSTOS DE ATENCIÓN}

Considerando los costos y unidades de los diversos conceptos descritos en el capítulo anterior, se estimó un costo directo de atención médica hospitalaria por paciente de USD 5803 en el HGZ N. 46 del IMSS, y de USD 11800 en el HR de PEMEX, que en función del número de pacientes atendidos en cada unidad médica, representó un costo total de USD 191512 para el IMSS y de USD 283195 para PEMEX, sumando entre ambas instituciones un monto total de USD 474707 (Tabla 2).

\section{DISCUSIÓN}

En esta serie se observó que la mayor frecuencia de fracturas de cadera en adultos mayores ocurrió en el grupo de 70 años y más en ambas instituciones. Este resultado es similar a lo reportado por Orces ${ }^{(12)}$ y por 


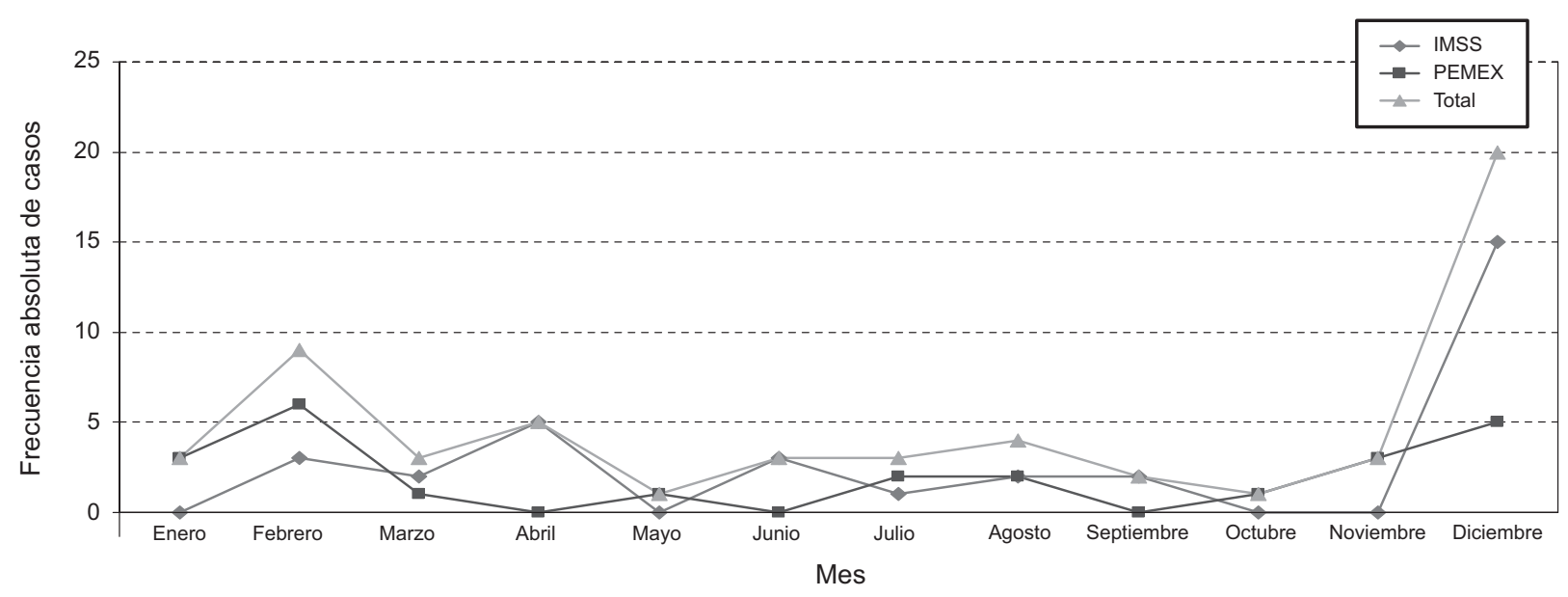

Figura 1. Casos de fractura de cadera de acuerdo al mes de ocurrencia. Fuente: Cálculo con base en informes institucionales.

Díaz ${ }^{(14)}$ quienes observaron que la edad promedio de ocurrencia de este fenómeno fue de 78 y 80 años, respectivamente. Esta similitud, puede atribuirse a que los adultos mayores de este grupo etario tienden a la fragilidad, lo que los coloca en situación de riesgo para caídas y otros síndromes geriátricos relacionados con esta condición. En lo que respecta al sexo, en concordancia con los autores ya citados ${ }^{(12,14)}$, se observó que la fractura de cadera se presentó con mayor frecuencia en el sexo femenino, presumiblemente, por los cambios en la densidad ósea que sufre este sexo, que aumenta la predisposición biológica a sufrir lesiones en este tejido.

En cuanto al tipo de fracturas, se observó que las más frecuentes fueron las del cuello del fémur (78,9\%), lo que difiere con lo reportado por Díaz ${ }^{(14)}$ quien informó haber observado que las fracturas más comunes son las trocantéreas (54\%) seguidas por las del cuello del fémur (45\%). Estas diferencias en cuanto al sitio de la lesión, pudieran estar relacionadas con el mecanismo del accidente o caída que la originó, sin embargo, esto deberá ser sometido a comprobación.

Con respecto a la temporalidad, se observó que la mayoría las fracturas de cadera se suscitaron en diciembre, lo que sugiere cierta estacionalidad de este padecimiento que al parecer no ha sido descrita ${ }^{(11-18)}$, por lo que deberá ser objeto de estudio en futuras investigaciones.

Por otra parte, con respecto a los costos estimados de atención al adulto mayor con fractura de cadera, considerando los diversos conceptos analizados en esta serie, se obtuvo un estimado de USD 5803 por paciente para el IMSS y de USD 11800 para PEMEX. Tales costos, pueden considerarse elevados al comparárseles con lo informado por Carlos ${ }^{(20)}$, quien obtuvo estimaciones de USD 1729,85 por paciente en el INR y de USD 3185,99 en el Seguro Popular. En ambos casos se trata de costos directos desde la perspectiva del financiador; no obstante, los resultados reportados por Carlos y cols. ${ }^{(20)}$, incluyen un mayor número de rubros, y aun así, el costo

Tabla 2. Costos directos (USD) de la atención de la fractura de cadera en el adulto mayor.

\begin{tabular}{|c|c|c|c|c|c|c|c|}
\hline \multirow[b]{2}{*}{ Concepto } & \multicolumn{3}{|c|}{ HGZ N. 46 - IMSS } & \multicolumn{3}{|c|}{ HR - PEMEX } & \multirow{2}{*}{$\begin{array}{l}\text { TOTAL } \\
(\mathrm{n}=57)\end{array}$} \\
\hline & $\begin{array}{c}\text { Costo } \\
\text { unitario }\end{array}$ & $\begin{array}{l}\text { Costo por } \\
\text { paciente }\end{array}$ & $\begin{array}{c}\text { Total } \\
(n=33)\end{array}$ & $\begin{array}{c}\text { Costo } \\
\text { unitario }\end{array}$ & $\begin{array}{l}\text { Costo por } \\
\text { paciente }\end{array}$ & $\begin{array}{c}\text { Total } \\
(n=24)\end{array}$ & \\
\hline Día de hospitalización & 407 & 3661 & 120818 & 554 & 4989 & 119743 & 240561 \\
\hline Honorarios médicos por día & 73 & 653 & 21534 & 177 & 1592 & 38213 & 59747 \\
\hline Intervención Quirúrgica & 1268 & 1268 & 41843 & 4755 & 4755 & 114125 & 155969 \\
\hline Laboratorio & 7 & 29 & 946 & 16 & 63 & 1523 & 2469 \\
\hline Radiodiagnóstico & 23 & 46 & 1509 & 54 & 107 & 2579 & 4088 \\
\hline Traslado en ambulancia & 147 & 147 & 4861 & 292 & 292 & 7012 & 11873 \\
\hline Total & - & 5803 & 191512 & - & 11800 & 283195 & 474707 \\
\hline
\end{tabular}

USD: dólares americanos; HGZ N. 46 - IMSS: Hospital General de Zona N. 46 del Instituto Mexicano del Seguro Social; HR - PEMEX: Hospital Regional de los Servicios Médicos de Petróleos Mexicanos 
observado en esta serie fue mucho mayor, especialmente en PEMEX. Estas cifras, representan un elevado gasto en materia de salud que debe ser regulado y estudiado a profundidad para ser estandarizado entre las instituciones del sector salud mexicano dadas las amplias divergencias existentes entre una institución y otra.

Asimismo, es pertinente mencionar las limitaciones y sesgos de la presente investigación. Con respecto a las limitaciones, la primera de ellas alude a los perfiles de los usuarios de las diversas instituciones prestadoras de servicios de salud, pues en cada caso, las características de los derechohabientes son en cierto sentido únicas y distintivas, tal es el caso del IMSS y de los Servicios Médicos de PEMEX, en donde el ingreso económico promedio mensual de una y otra población constituye una marcada diferencia (21); situación por la que los resultados del presente estudio, en cuanto a toma de decisiones, son solo aplicables a las instituciones aludidas. Sin embargo, pueden ser considerados como parámetro de referencia para futuros estudios en otras instituciones y latitudes. El sesgo que marca principalmente a esta investigación es el de observación, pues en este estudio el registro de casos y costos fue retrospectivo y hubo elementos que escaparon del análisis realizado. En los conceptos incluidos para la estimación de los costos, por ejemplo, estos se limitan a la atención quirúrgica de la fractura de cadera, dejando de lado otros conceptos como la estancia intrahospitalaria previa a la atención quirúrgica, el tipo de material de osteosíntesis utilizado, el número de estudios de radiodiagnóstico realizados de forma transoperatoria, las consultas ambulatorias de especialidad para el seguimiento del paciente, el costo de la reintervención para el retiro de material de osteosíntesis en caso de ser requerido (con su consecuente estadía intrahospitalaria), entre otros aspectos, así como la posibilidad de que el proceso de atención se vea afectado por variables que prolonguen y aumenten los costos de atención, como las complicaciones pre y postoperatorias.

En conclusión, el número de adultos mayores hospitalizados durante el año 2009 en las dos instituciones objeto de estudio fue elevado, especialmente en el HR de PEMEX. La prevalencia de fractura de cadera en los adultos mayores hospitalizados en el HGZ N. ${ }^{\circ} 46$ del IMSS y el HR de PEMEX en el año 2009, se puede presumir baja ante la carencia de reportes contra los cuales compararlas, no obstante, entre estas dos instituciones, la prevalencia fue mayor en el IMSS. Las fracturas de cadera se observan con mayor frecuencia en adultos mayores de 70 años, sexo femenino. Existe la posibilidad de que haya un comportamiento estacional en la frecuencia de los casos, lo que constituiría una oportunidad de intervención. El costo directo estimado de atención hospitalaria a los adultos mayores con fractura de cadera, fue mayor a lo informado en la literatura nacional consultada en término unitarios.

Se requieren estudios posteriores de mayor amplitud para definir con claridad si existe comportamiento estacional de la fractura de cadera y diseñar en consecuencia estrategias para la reducción de su frecuencia. Asimismo, se requieren estudios prospectivos en los que se lleve a cabo un seguimiento estricto de los costos de atención para definir el costo real de la atención y ofrecer de éste modo un diagnóstico situacional $100 \%$ fidedigno en lugar de una estimación, incluyendo además de este, el origen de la lesión.

\section{AGRADECIMIENTOS}

A las autoridades del Hospital General de Zona N. ${ }^{\circ} 46$ del IMSS y del Hospital Regional de PEMEX, en Villahermosa, Tabasco, México, por las facilidades otorgadas para el acceso a la información con la que fue elaborado el presente trabajo de investigación.

\section{Contribuciones de autoría}

QTEC, ZGMA, HGAC y HOHM participaron en la concepción y diseño del trabajo. ZGMA, HGAC y HOHM participaron en la recolección de información. ZGMA participó en el análisis e interpretación de datos. ZGMA, HGAC y HOHM participaron en la redacción de la primera versión del manuscrito. QTEC y ZGMA participaron en la redacción de la segunda versión del manuscrito. Todos los autores revisaron críticamente versiones preliminares y aprobaron la versión final.

\section{Fuentes de financiamiento}

Autofinanciado.

\section{Conflictos de interés}

Los autores declaran no tener conflictos de interés en la publicación de este artículo.

\section{REFERENCIAS BIBLIOGRÁFICAS}

1. Muñoz $O$, García-Peña $C$, Durán $L$ (eds). La salud del adulto mayor. Temas y debates. México: Centro Interamericano de Estudios de Seguridad Social / Instituto Mexicano de Seguro Social; 2004.

2. Consejo Nacional de Población (CONAPO). El envejecimiento de la población en México. México: CONAPO; 2005.

3. Wong R, Espinoza M, Palloni A. Adultos mayores mexicanos en contexto socioeconómico amplio: Salud y envejecimiento. Salud Publica Mex. 2007;49(Suppl4):S436-47.

4. Consejo Nacional de Población (CONAPO). Proyecciones de población municipales 2005-2050. México: CONAPO; 2005. 
5. Sánchez-González D. Envejecimiento demográfico urbano y sus repercusiones socio-espaciales en México. Retos de planeación gerontológica. Rev Geografía Norte Grande. 2007;(38):45-61.

6. Zuñiga-Herrera E. Tendencias y características del envejecimiento demográfico en México. En: Consejo Nacional de Población (CONAPO). Situación demográfica en México, 2004. México: CONAPO; 2005.

7. Rodríguez R, Morales J, Encinos J, Trujillo Z, D’hyver C. Geriatría. México: McGraw-Hill Interamericana; 2000. p. 187-230.

8. da Silva ZA, Gómez-Conesa A, Sobral-Ferreira M. Epidemiología de caídas de ancianos en España. Una revisión sistémica, 2007. Rev Esp Salud Publica. 2008;82(1):43-55.

9. Stevens JA, Ryan G, Kresow M. Fatalites and injuries from falls among older adults. Unite States, 1993-2003 and 2001-2005. MMWR. 2006;55(45):1221-4.

10. Cotter PE, Timmons S, Tworney C, O'Mahony D. The financial implications of falls in older people for an acute hospital. Ir J Med Sci. 2006;175(2):11-3.

11. Martinez-Rondanelli A. Fractura de cadera en ancianos: Pronósticos, epidemiología. Aspectos generales. Experiencia. Rev Col de Or Tra. 2005;19(1):20-8.

12. Orces $\mathbf{C H}$. Epidemiology of hip fractures in Ecuador. Rev Panam Salud publica/Pan Am J Public Health. 2009;25(5):438-42.

13. Jaatinen PT, Panula J, Aarnio P, Kievelä SL. Incidence of hip fractures among the ederly in Satakunta, Finland. Scand J Surg. 2007;96:256-60.

14. Díaz A, Curto JM, Ferrandez L. Epidemiología de las fracturas de la extremidad superior del fémur. Rev Esp Cir Osteoart. 1993;28:267-70.

15. Marks R. Hip fracture epidemiological trends, outcomes, and risk factors, 1970-2009. Int J Gen Med. 2010;3:1-17.

16. Hye-young $K$. Incidence and mortality of hip fracture among the elderly population in South Korea: A populationbased study using the National Health Insurance claims data. BMC Public Health. 2010;10(230).

17. Öztürk A, Özkan Y, Akgõz S, Yalçýn N, Özdemir RM, Aykut S. The risk factors of mortality in elderly patients with hip fractures postoperative one-year results. Singapure Med J. 2010;51(2):137-43.
18. Julieb V, Krogseth M, Skovlund E, Engedal K, Wyller T. Medical treatment predicts mortality after hip fracture. J Gerontol A Biol Sci Med Sci. 2010;65A(4):442-9.

19. Orosz GM, Magaziner J, Hannan EL, Morrison RS, Koval K, Gilbert M, et al. Association of timing of surgery for hip fracture and patient outcomes. JAMA. 2004;291(14):1738-43.

20. Carlos F, Clark P, Maciel H, Tamayo JA. Direct costs of osteoporosis and hip fracture: An analysis for the Mexican Social Insurance Health Care System. Salud Publica Mex. 2009;51(Suppl1):S108-13.

21. Instituto Nacional de Estadística, Geografía e Informática (INEGI). Perspectiva estadística. Tabasco. Junio 2011. México: INEGI; 2011

22. Instituto Mexicano del Seguro Social (IMSS). Acuerdo ACDO.SA3.HCT.240310/57.P.DF del H. Consejo Técnico por el que se aprueban los costos unitarios por nivel de atención médica para el año 2010 y la base de cálculo para la actualización de los costos unitarios por nivel de atención médica para el año 2010. México: Diario Oficial de la Federación; 2010.

23. Petróleos Mexicanos (PEMEX). Acuerdo PA-801-78300-4 de la Gerencia de Administración y Finanzas de la Subdirección de Servicios de Salud de la Dirección Corporativa de Administración, por el que se aprueba la actualización de cuotas por atención médica para el año 2009. México: PEMEX; 2009.

24. Centro Nacional de Excelencia Tecnológica en Salud (CENETEC-SALUD). Guía de práctica clínica. Diagnóstico y tratamiento de fracturas intraescapulares del extremo proximal del fémur. México: CENETEC-SALUD; 2009.

Correspondencia: Marco Antonio Zavala González Dirección: Calle principal de la alberca \# 24, Col. Ingenio Santa Rosalía, H. Cárdenas, Tabasco, México, C.P. 86470. Teléfono: +52 (937) 1264197

Correo electrónico: zgma_51083@yahoo.com.mx

Visite los contenidos de la revista en: www.ins.gob.pe/rpmesp 